2-1-2019

\title{
Analysis of Oxygen-18 Labeled Phosphate to Study Positional Isotope Experiments Using LC-QTOF-MS
}

\author{
Sujatha Chilakala \\ Cleveland State University \\ Iteen Cheng \\ Case Western Reserve University \\ Ireen Lee \\ Case Western Reserve University \\ Yan Xu \\ Cleveland State University, y.xu@csuohio.edu
}

Follow this and additional works at: https://engagedscholarship.csuohio.edu/scichem_facpub

Part of the Analytical Chemistry Commons, and the Biochemistry Commons

How does access to this work benefit you? Let us know!

\section{Recommended Citation}

Chilakala, Sujatha; Cheng, Iteen; Lee, Ireen; and Xu, Yan, "Analysis of Oxygen-18 Labeled Phosphate to Study Positional Isotope Experiments Using LC-QTOF-MS" (2019). Chemistry Faculty Publications. 508. https://engagedscholarship.csuohio.edu/scichem_facpub/508

This Article is brought to you for free and open access by the Chemistry Department at EngagedScholarship@CSU. It has been accepted for inclusion in Chemistry Faculty Publications by an authorized administrator of EngagedScholarship@CSU. For more information, please contact library.es@csuohio.edu. 


\title{
Analysis of oxygen-18 labeled phosphate to study positional isotope experiments using LC-QTOF-MS
}

\author{
Sujatha Chilakala ${ }^{a}$, Iteen Cheng', Ireen Lee ${ }^{\mathrm{b}}$, Yan $\mathrm{Xu}^{\mathrm{a}}{ }^{\mathrm{k},}$ \\ ${ }^{a}$ Department of Chemistry, Cleveland State University, 2121 Euclid Avenue, Cleveland, OH, 44115, USA \\ ${ }^{b}$ Department of Chemistry, Case Western Reserve University, Cleveland, OH, 44106, USA
}

A R T I C L E I N F O

Keywords:

Positional isotope exchange

O-18 labeled phosphate

LC-QTOF-MS

ATP hydrolysis

${ }^{18} \mathrm{O} /{ }^{16} \mathrm{O}$ ratio

\begin{abstract}
A B S T R A C T
A method is proposed in this paper for the determination of oxygen-18 labeled phosphate so that positional isotope experiments using sensitive and rapid liquid chromatography-QTOF-mass spectrometry (LC-QTOF-MS) experiments can be carried out. The positional isotope exchange technique is a useful tool in understanding the mechanisms and kinetics of many enzyme-catalyzed reactions. Detection of the positions and concentration of these exchanged isotopes is the key. Gas chromatography-mass spectrometry (GC-MS) and nuclear magnetic resonance imaging are commonly used analytical techniques for measurement of ${ }^{18} \mathrm{O} /{ }^{16} \mathrm{O},{ }^{31} \mathrm{P}$ and ${ }^{15} \mathrm{~N}$ isotope enrichment. Since these techniques either require a time-consuming derivatization step or have a limited sensitivity, an LC and accurate mass-based method for monitoring ${ }^{18} \mathrm{O} /{ }^{16} \mathrm{O}$ exchange was developed and compared with a standard GC-MS method. Our results showed that the LC-QTOF-MS method developed was not only as accurate as the standard GC-MS method, but also a sensitive and robust analytical platform for the simultaneous determination of isotope enrichment and the analysis of positional isotopes without chemical derivation. The LCQTOF-MS method developed was successfully applied to the measurement of ${ }^{18} \mathrm{O} /{ }^{16} \mathrm{O}$ in the reversibility study of ATP hydrolysis by Lon proteases.
\end{abstract}

\section{Introduction}

The positional isotope exchange (PIX) technique has proven to be a valuable tool for both the identification of reaction intermediates and reaction reversibility and in the elucidation of the mechanistic pathways of many enzyme-catalyzed reactions $[1,2]$. The general approach involves reactions being conducted in an isotope-enriched buffer solution so that the extent of the incorporation of the isotopes into the intermediates or products generated by the enzymatic reactions is used to evaluate the kinetics of the reaction or the extent of the reversibility process. PIX techniques have also been used in time course studies to calculate individual rate constants or the ratio of rate constants in a reaction mechanism [3-5]. The most commonly used PIX technique for enzyme-catalyzed reactions involves phosphate and the transfer of oxygen. In oxygen transfer PIX reactions, labeled oxygen $\left({ }^{18} \mathrm{O}\right)$ is exchanged with the oxygen bonded to an inorganic phosphate, and the measurement of the ${ }^{18} \mathrm{O} /{ }^{16} \mathrm{O}$ ratio is used to determine the reaction mechanism and kinetics.

The most commonly used method for detecting ${ }^{18} \mathrm{O} /{ }^{16} \mathrm{O}$ isotope enrichment involves gas chromatography-mass spectrometry (GC-MS) of a derivatised form of the product. Nuclear magnetic resonance
(NMR) imaging involving ${ }^{31} \mathrm{P}$ and ${ }^{15} \mathrm{~N}$ [6-8] has been used to study isotope enrichment utilizing labeled phosphate and nitrogen. The main disadvantage of GC-MS is that a volatile derivative of the isotope labeled compound needs to be prepared for analysis [9,10]. However, although ${ }^{31} \mathrm{P}$ NMR imaging offers a direct analysis method, its sensitivity is insufficient and the process is time-consuming $[11,12]$.

Although oxygen labeled isotope exchange reactions have been widely used to monitor the reversibility of enzyme reactions and their kinetics [6], no sensitive and simple analytical method has been developed for the analysis of ${ }^{18} \mathrm{O}$-labeled phosphate in PIX experiments. In order to address this shortcoming, this study proposes a simple, sensitive, and efficient LC-QTOF-MS method for studying ${ }^{18} \mathrm{O}$ isotope enrichment in inorganic phosphate in PIX experiments. The accurate mass spectroscopy of this method allows for both labeled and unlabeled phosphate ions to be specifically detected, and this can be achieved at resolutions of over 20,000 .

In this study, we demonstrate that this new method can be used to detect the reversibility of the adenosine triphosphate (ATP) hydrolysis of Lon proteases. Lon is an ATP-dependent serine protease. It is known that ATP hydrolysis increases the protein hydrolytic activity of Lon $[13,14]$. Despite being a homo-oligomer, Lon has a set of high- and low- 
affinity sites for ATP, which suggests that there is a functional nonequivalency in the sub-units of Lon. This half-site reactivity has already been demonstrated by chemical quench flow experiments, which have allowed for the initial turnover of ATP hydrolysis at different ATPase sites in Lon to be monitored. A reactivity of only $50 \%$ was observed in these experiments [13,14]; this supported the half-site reactivity theory, in which identical ATPase active sites on each Lon sub-unit possess two different affinities for ATP. In order to confirm that the $50 \%$ reactivity is due to the half-site reactivity and not the reversibility of ATP hydrolysis, and in order to better understand how ATP hydrolysis contributes to the enzyme catalysis of peptide bond breakages, we must determine if ATP hydrolysis is reversible in Lon. If it is, then one ATP molecule may be responsible for repeated rounds of protease activity, because the molecule can continuously regenerate. This reversibility can be detected by adding ${ }^{18} \mathrm{O}$-labeled water to an ATPase reaction mixture, as this allows for PIX to occur within phosphate molecules; we can then monitor the number of labeled oxygen atoms incorporated in the resultant inorganic phosphate by using mass spectrometry [13-15].

The data we obtained using LC-QTOF-MS was compared to data obtained using GC-MS, and they were found to be in good agreement. Our proposed method is therefore excellent for analyzing the isotope enrichment produced by PIX experiments during the study of enzyme reactions.

\section{Experimental}

\subsection{Chemicals and materials}

Ammonium formate, water with 25 atom $\%{ }^{18} \mathrm{O}$, N-trimethylsilyldiethylamine (TMSDEA) (95\%), dithiothreitol, magnesium acetate, formic acid, and ethylenediaminetetraacetic acid (EDTA) were procured from Sigma-Aldrich (St. Louis, MO, USA). Phosphoric acid (85.0\%) and hydrochloric acid $(38.0 \%)$ were procured from Fischer Scientific (Hanover park, IL, USA). Deionized water (resistivity $=18.2 \mathrm{M \Omega} \cdot \mathrm{cm}$ ) was prepared using a Barnstead Model 7148 Nanopure ultrapure water system from Thermo Scientific (Waltham, MA, USA).

\subsection{Sample preparation}

For the analysis of the isotope enrichment performed by the LCQTOF-MS method, standard working solutions (10.0, 50.0, 100, 250, 500 , and $1.00 \times 10^{3} \mathrm{nM}$ ) of phosphoric acid were prepared by diluting and mixing appropriate volumes of concentrated phosphoric acid $\left(\mathrm{H}_{3} \mathrm{PO}_{4}, 85 \%\right)(14.62 \mathrm{M})$ with deionized water.

A stock solution of ammonium formate $(0.10 \mathrm{M})$ was prepared by dissolving an appropriate amount of ammonium formate salt in a known volume of HPLC-grade water and storing it in a refrigerator at $4{ }^{\circ} \mathrm{C}$ before use. A working solution of ammonium formate $(10.0 \mathrm{mM})$ was then prepared through a ten-fold dilution of the stock solution with HPLCgrade water; the $\mathrm{pH}$ was adjusted to 3.65 using formic acid, if necessary.

For the GC-MS analysis, samples were prepared and derivatised as described in earlier studies $[6,13,15]$; in brief, $10.0 \mathrm{~nL}$ of phosphoric acid (ca. $500 \mathrm{nM}$ in methylene chloride) was transferred together with $10.0 \mathrm{~nL}$ of TMSDEA into a $1.5 \mathrm{~mL}$ glass vial (VWR, Radnor, PA, USA). After the vial cap had been tightened, a derivatization reaction was allowed to proceed at room temperature for $60 \mathrm{~min}$ with occasional shaking. The derivatization of the inorganic phosphate with TMSDEA generated tris(trimethylsilyl)phosphate (TMSP). The final product was analyzed using GC-MS. The derivatised samples were stored at $-20{ }^{\circ} \mathrm{C}$ until the GC-MS analysis was performed.

\subsection{Instrumentation}

\subsubsection{LC-QTOF-MS chromatography separation and mass spectrometric conditions}

The LC-QTOFMS system used in this work consisted of an Agilent
Infinity 1290 UPLC system that was interfaced with an Agilent QTOF 6540 mass spectrometer (Santa Clara, CA, USA). Agilent Infinity 1290 UPLC is composed of a solvent reservoir, a degasser, a binary pump (G4220A), a thermostat (G1330B), an autosampler (G4226A), and a thermostatted column compartment (G1316C). The quadrupole timeof-flight (Q-TOF 6540) mass spectrometer was equipped with an orthogonal ESI-interfaced Agilent Jet Stream (AJS) operated under a negative mode; the data was acquired in a data-dependent manner. The instrument was controlled by a personal computer running the Mass Hunter Workstation (version B.05.01) software from Agilent Technologies (Santa Clara, CA, USA).

The LC separation was carried out on Acclaim Trinity P2 $(100 \times 2.1 \mathrm{~mm} ; 3.6 \mathrm{~nm}$ particle size $)$ from Thermo Fisher Scientific (Waltham, MA, USA) maintained at $30^{\circ} \mathrm{C}$ with a mobile phase containing $25 \%$ acetonitrile and $75 \% 10.0 \mathrm{mM}$ ammonium formate with a $\mathrm{pH}$ of 3.65 at a flow rate of $0.3 \mathrm{~mL} / \mathrm{min}$. Prior to the initial sample analysis, the column was equilibrated with at least 20 column volumes of the mobile phase. For each run, $10.0 \mu \mathrm{L}$ of the sample was injected into the system by an autosampler that was set to $4.0^{\circ} \mathrm{C}$.

The mass spectrometric detection was carried out using the negative electrospray ionization (ESI-) mode, which was tuned for compounddependent and source-dependent parameters. The optimized mass spectrometry operating parameters were as follows: capillary voltage, $2250 \mathrm{~V}$; nebulizer pressure, 40 psi; drying gas flow rate, $10 \mathrm{~L} / \mathrm{min}$; gas temperature, $350{ }^{\circ} \mathrm{C}$; sheath gas flow, $11 \mathrm{~L} / \mathrm{min}$; sheath gas temperature, $325^{\circ} \mathrm{C}$; skimmer voltage, $65 \mathrm{~V}$; and fragment voltage, $150 \mathrm{~V}$. Both the centroid and profile modes were used in the data collection with an extended dynamic range of $2 \mathrm{GHz}$. MS accurate mass spectra were recorded for $90-110 \mathrm{~m} / \mathrm{z}$ at a speed of 5 spectra/s, The TOF was calibrated on a daily basis, and a real-time calibration was performed by the continuous infusion of reference ion 121.0508.

\subsubsection{GC-MS chromatography separation and mass spectrometric} conditions

The GC-MS system used in this work consisted of a Varian gas chromatograph interfaced with a Varian Saturn 2100T ion trap mass spectrometer (Agilent Technologies, Santa Clara, CA, USA). The GC separation was carried out using an Agilent VF5-MS GC column $(30 \mathrm{~m} \times 0.25 \mathrm{~mm} \times 0.2 \mathrm{~nm})$ in which helium was used as a carrier gas at a constant flow rate of $1.00 \mathrm{~mL} / \mathrm{min}$. In each run, a $1.00 \mathrm{~nL}$ sample was injected with a split ratio of $1: 10$, and the injector temperature was maintained at $250^{\circ} \mathrm{C}$. The temperature profile was begun at $60^{\circ} \mathrm{C}$ before being increased to $110^{\circ} \mathrm{C}$ at a rate of $20^{\circ} \mathrm{C} / \mathrm{min}$; following this, it was increased to $240{ }^{\circ} \mathrm{C}$ at a rate of $40{ }^{\circ} \mathrm{C} / \mathrm{min}$ before being held at this temperature for $5 \mathrm{~min}$.

The electron impact (EI) ionization mode was used for the MS detection. The optimized mass spectrometry operating parameters were as follows: target total ion count, 11000 counts; maximum ionization time, $25000 \mathrm{~ns}$; and pre-scan ionization time, $100 \mathrm{~ns}$ The mass spectra were obtained in the mass range of $m / z 100-400$. The following extracted ion chromatograms of the TMSP ions (including the parent ion) were monitored: $\mathrm{TMSP}^{16} \mathrm{O}_{4}-\mathrm{CH} 3$ (299), parent ion $+2 \mathrm{~m} / \mathrm{z}$ : $\mathrm{TMSP}^{16} \mathrm{O}_{3}{ }^{18} \mathrm{O}-\mathrm{CH} 3$ (301), and parent ion $+4 \mathrm{~m} / \mathrm{z}$ : $\mathrm{TMSP}^{16} \mathrm{O}_{2}{ }^{18} \mathrm{O}_{2}-\mathrm{CH} 3$ (303).

\subsection{Isotope enrichment calculation}

The chromatograph peaks were extracted using the accurate mass of each ion species (i.e., the extracted ion chromatograms), and the isotope enrichment achieved by the experiment was calculated using the following equation [6]:

relative \% isotope $=\frac{\text { isotope peak signal }}{\text { signal of primary isotope }} \times 100$

In order to correctly account for the enrichment due to ${ }^{18} \mathrm{O}$ from the data obtained from the LG-QTOF-MS analysis, the expected natural isotopic abundance was subtracted from the experimental abundance. 
For the data obtained using GC-MS, the isotopic spillover was calculated; this was because the derivatization reagent TMSP has a high natural abundance of ${ }^{29} \mathrm{Si}$ and ${ }^{30} \mathrm{Si}$, which can obscure the interpretation of the ${ }^{18} \mathrm{O}$ incorporation results. This isotopic spillover was calculated as per the method described in a previously published paper [6]. In order to correctly account for the enrichment due to ${ }^{18} \mathrm{O}$, the expected natural isotopic abundance was subtracted from the experimental abundance. The spillover from the species was subtracted from the ${ }^{29} \mathrm{Si}$ and ${ }^{30} \mathrm{Si}$ isotopes in addition to the expected natural abundance of ${ }^{18} \mathrm{O}$.

\subsection{PIX with the Lon protease}

The PIX reactions with the Lon protease were carried out to study reversibility of ATP hydrolysis by LON proteases in the presence and in the absence of the substrate peptide as per the method described in previously published papers $[13,15]$. All the samples were prepared in triplicate with two control experiments. The first control experiment contains only inorganic phosphate (phosphoric acid) without enzymes being added. In the second control experiment, ATP was incubated in the reaction mixture without enzymes being added. The samples were then prepared for each LC-QTOF-MS and GC-MS analysis separately, as described in the sub-sections above.

\section{Results and discussion}

\subsection{Method development}

\subsubsection{LC-QTOF-MS method}

In this work, a QTOF-MS) was used for analyte detection due to its high mass accuracy and resolution. Because the inorganic phosphate readily deprotonates in the acidic mobile phase ( $\mathrm{pH} \mathrm{3.65),} \mathrm{ESI} \mathrm{-} \mathrm{was}$ chosen as the means of sample introduction. In order to demonstrate the utility of the LC-MS method for the detection of PIX in enzymecatalyzed reactions, phosphoric acid $(100 \mathrm{nM})$ was used in this method. The predominant ion for the unlabeled sample in the negative ion mode occurs at $\mathrm{m} / \mathrm{z} 96.9896$ for the parent ion $\left(\mathrm{H}_{2} \mathrm{PO}_{4}{ }^{-}\right)$species. Isotope peaks were also observed at parent ion $+2 \mathrm{~m} / \mathrm{z}$ : 98.9864 $\left(\mathrm{H}_{2} \mathrm{P}^{16} \mathrm{O}_{3}{ }^{18} \mathrm{O}^{-}\right)$and parent ion $+4 \mathrm{~m} / \mathrm{z}$ : $100.9972\left(\mathrm{H}_{2} \mathrm{P}^{16} \mathrm{O}_{2}{ }^{18} \mathrm{O}_{2}{ }^{-}\right)$.

Due to the ionic nature of the analyte, which is difficult to retain in reverse phase LC columns without the use of an ion pair agent, ion exchange chromatographic columns were considered for the analytical separation. Several columns, including Waters HILIC (Waters Corporation, Milford, MA, USA) and Thermo Fisher Scientific Acclaim Trinity P2, were tested. The Acclaim Trinity P2 column was chosen from these, because it provides hydrophilic interaction chromatography (HILIC) and a strong anion-exchange mixed-mode retention mechanism, and it exhibited more symmetrical and sharper peaks with better sensitivity and signal-to-noise ratio than the HILIC column.

The composition of the mobile phase used for the separation was optimized. Acetonitrile was chosen as the organic solvent due to its greater solvent strength and the higher signal response of analytes in it during mass spectrometry than could be obtained using methanol. Another reason as to why it was preferred over methanol was due to the presence of a carboxylic acid group in the stationary phase of the column. The percentage content of acetonitrile in the mobile phase was also investigated. When a greater amount $(>40 \%)$ of acetonitrile was used, the analyte was not retained in the column. Furthermore, because the $\mathrm{pH}$ value of the mobile phase influences the ionization and retention of phosphoric acid, the optimal $\mathrm{pH}$ of the mobile phase was investigated. In this study, the baseline resolution of the analyte was achieved with ammonium formate $(10.0 \mathrm{mM})$ at $\mathrm{pH} 3.65$. At this $\mathrm{pH}$ value, phosphoric acid $\left(\mathrm{PKa}_{1}=2.15\right)$ was in its deprotonated form.

In this study, the optimized liquid chromatographic separation was obtained using a Thermo Fisher Scientific Acclaim Trinity P2 C18 column and a mobile phase consisting of $25 \%$ acetonitrile and $75 \%$
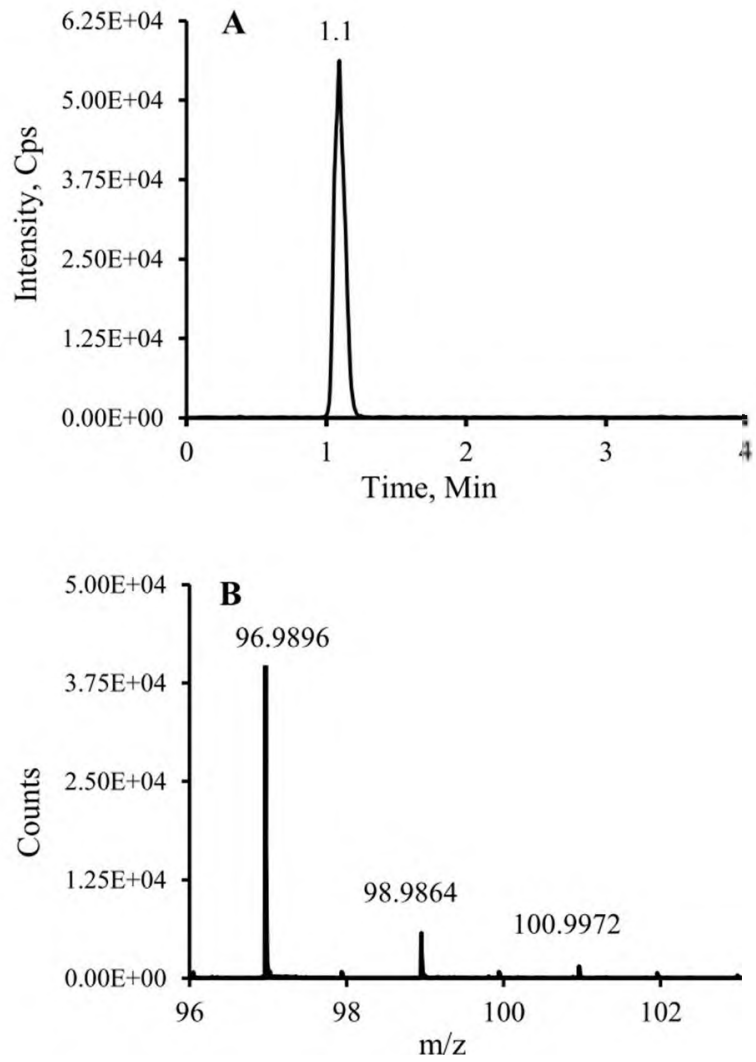

Fig. 1. Determination of the ${ }^{18} \mathrm{O} /{ }^{16} \mathrm{O}$ ratios using LC-QTOF-MS. (a) Representative total ion chromatogram of inorganic phosphate and (b) representative mass spectral resolution of the phosphate isotopologues.

$10.0 \mathrm{mM}$ ammonium formate pumped at a flow rate of $0.300 \mathrm{~mL} / \mathrm{min}$. The total run time was $4.00 \mathrm{~min}$, with a retention time of $1.2 \mathrm{~min}$. Fig. 1(a) and (b) show the representative total ion chromatogram and mass spectra of the sample, respectively. The relative isotope amounts were calculated using Eq. (1). A linear calibration curve was created with phosphoric acid standard solutions ranging from 10.0 to $1.00 \times 10^{3} \mathrm{nM}$ (Fig. 2).

\subsubsection{GC-MS method}

The GC-MS analysis was carried out as per the method described in a previously published paper $[13,15]$. The GC separation was carried out using an Agilent VF5-MS GC column. The isotope enrichment was analyzed using the extracted ion chromatogram (EIC) mode of the iontrap mass spectrometer. Fig. 3 shows the representative extracted ion

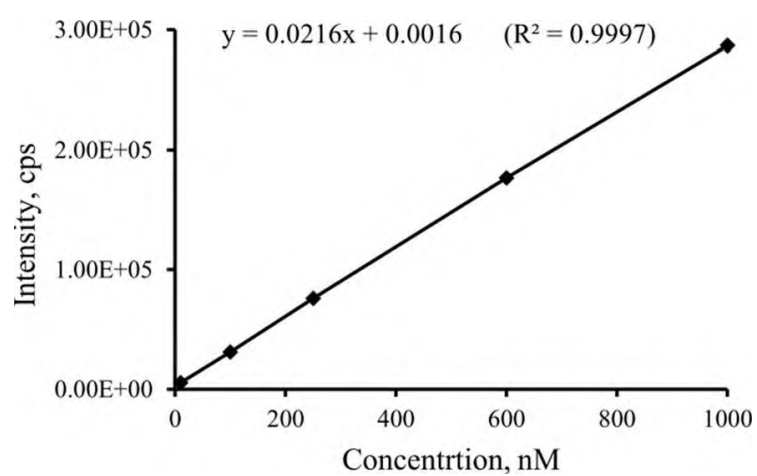

Fig. 2. Calibration curve established using the phosphoric acid standard solutions from 10.0 to $1.00 \times 10^{3} \mathrm{nM}$ using the LC-QTOF-MS method. 


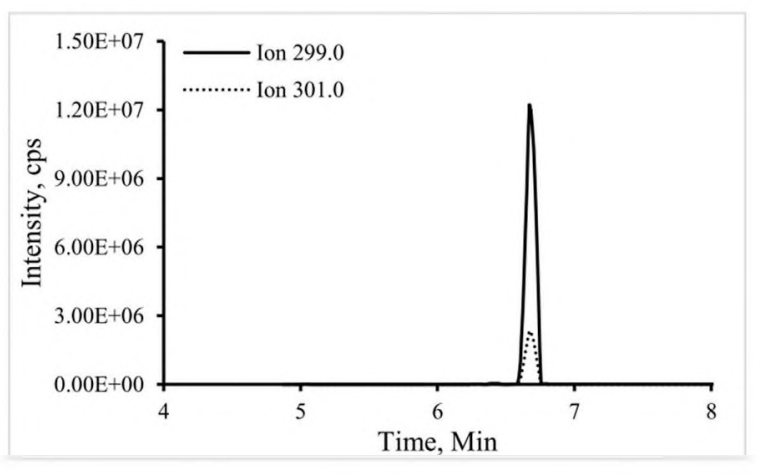

Fig. 3. Representative selected ion chromatograms of TMSP used for the determination of the ${ }^{18} \mathrm{O} /{ }^{16} \mathrm{O}$ ratios in inorganic phosphate using GC-MS.

chromatograms of the parent ion $=\mathrm{TMSPO}_{4}-\mathrm{CH} 3$ (299.0) and parent ion $+2 \mathrm{~m} / \mathrm{z}=\mathrm{TMSP}^{16} \mathrm{O}_{3}{ }^{18} \mathrm{O}-\mathrm{CH} 3(301.0)$ from the GC-MS analysis.

\subsection{Application}

\subsubsection{PIX with the Lon protease}

In order to demonstrate the applicability of the LC-MS method, we monitored the isotope enrichment in PIX reactions catalyzed by ATPase in the presence and absence of a peptide substrate $[13,15]$. In brief, in these reactions ADP is produced by ATP hydrolysis with the Lon protease, which is a rate-limiting step that releases inorganic phosphate as a by-product. In order to check the reversibility of the ATPase reactions in the presence and absence of this peptide substrate, the experiments were conducted in ${ }^{18} \mathrm{O}$-enriched water so that the number of labeled oxygen atoms incorporated in the resultant inorganic phosphate generated by the ATP hydrolysis could be monitored. Furthermore, in this study, inorganic phosphate was used as a positive control, and the reaction mixture without the enzyme was used as a negative control.

In the LC-QTOF-MS analysis, the lyophilized inorganic phosphate samples were prepared by being dissolved in deionized water. For the GC-MS analysis, the inorganic phosphate product was converted to a volatile derivative by the reaction with TMSDEA; the extent of the incorporation of ${ }^{18} \mathrm{O}$ was thusly determined by GC-MS. In order to ascertain if the ATP hydrolysis was a reversible process, we determined the number of isotopically labeled oxygen atoms incorporated into the phosphate from an enriched reaction mixture by comparing it to the natural isotopic abundance. Furthermore, control experiments were performed by incubating ATP in the reaction mixture without enzymes being added, so that we could ensure that no labeled oxygen was incorporated due to the non-enzymatic hydrolysis during the time course of the experiment.

Additional isotopically labeled oxygen atoms will be detected if the phosphate combines with ADP in the reverse reaction and unlabeled oxygen is lost due to dehydration. As a result, detection of more than one incorporation of ${ }^{18} \mathrm{O}$ into the inorganic phosphate reveals if the ATP hydrolysis is reversible at the ATPase sites. Fig. 4(a) and (b) show the results of the isotope enrichment experiments from both the LCQTOF-MS and GC-MS analyses, respectively. As can be seen in Fig. 4, in the presence of the peptide substrate, we detected enrichment in the parent ion $+2 \mathrm{~m} / \mathrm{z}\left(\mathrm{H}_{2} \mathrm{P}^{16} \mathrm{O}_{3}{ }^{18} \mathrm{O}^{-}\right)$; namely, an enrichment in $\left(\mathrm{H}_{2} \mathrm{P}^{16} \mathrm{O}_{3}{ }^{18} \mathrm{O}^{-}\right)$of $21 \pm 3 \%$ over the expected natural abundance. In the absence of the peptide substrate, an enrichment of $2.5 \pm 1 \%$ in the parent ion $+2 \mathrm{~m} / \mathrm{z}\left(\mathrm{H}_{2} \mathrm{P}^{16} \mathrm{O}_{3}{ }^{18} \mathrm{O}^{-}\right)$was observed. The data from the LC-QTOF-MS was consistent with the GC-MS data, which means that both methods could be used for such studies.

In order to further analyze the usefulness of the method for studying the time course of peptide hydrolyses, we measured the isotope enrichment in the inorganic phosphate. The PIX reactions with the Lon protease were carried out for $0,5,10,30$, and $60 \mathrm{~min}$ (as described in Section 2.5). As shown in Fig. 5, no enrichment was observed in the control. Much less
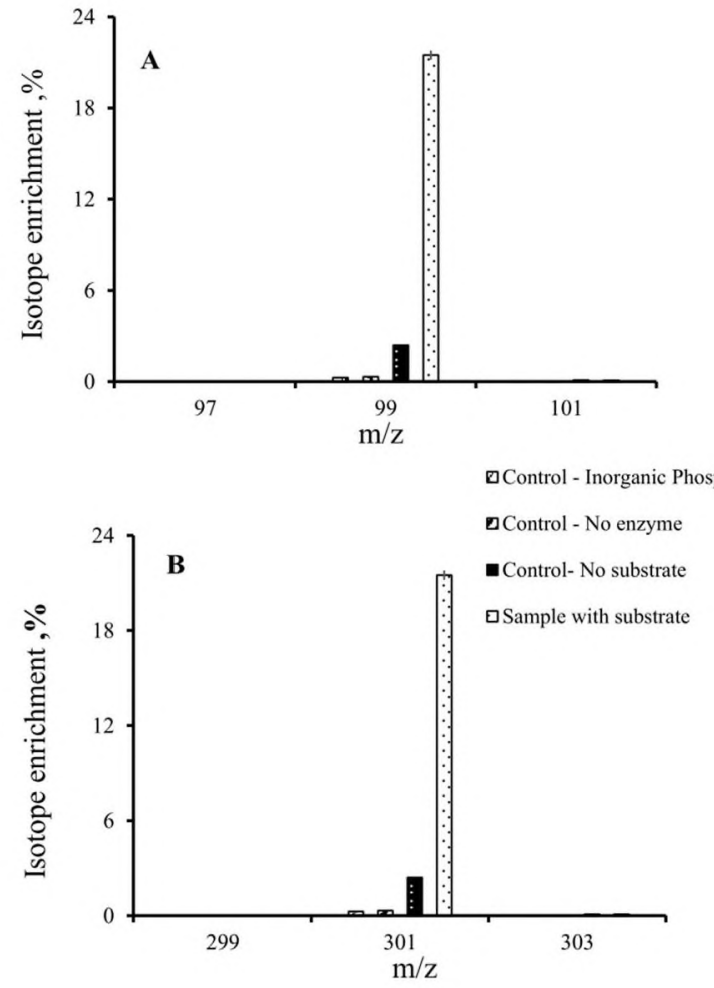

Fig. 4. Isotope enrichment calculated for the incorporation of ${ }^{18} \mathrm{O}$ into the inorganic phosphate released from the hydrolysis of ATPase in the presence of isotopically enriched water. (a) Enrichment measured using the LC-QTOF-MS method and (b) enrichment measured using the GC-MS method.

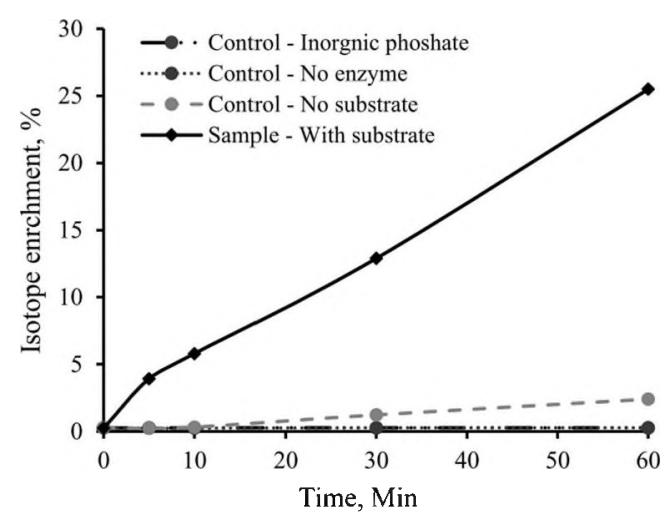

Fig. 5. Time study analysis of isotope enrichment for the incorporation of ${ }^{18} \mathrm{O}$ into the inorganic phosphate from the hydrolysis of ATP by the Lon protease using the LC-QTOF-MS method.

enrichment was observed in the reaction mixture that did not contain the peptide substrate than the one that did contain it.

\section{Discussion}

A number of methods like GC-MS and NMR have been utilized for the analysis of PIX rates in enzyme-catalyzed reactions. Although GCMS methods are widely used for the analysis of PIX experiments, the main disadvantage of such methods is the need for the chemical derivatization of the samples so as to make them volatile; however, this is time-consuming (i.e., it takes $1 \mathrm{~h}$ to do). Furthermore, the derivatization reagent TMSP has a high natural abundance of ${ }^{29} \mathrm{Si}$ and ${ }^{30} \mathrm{Si}$, which can obscure the interpretation of the results of the incorporation of ${ }^{18} \mathrm{O}$ if left unchecked [14]. In order to correctly account for the enrichment 
due to ${ }^{18} \mathrm{O}$, the expected isotopic abundance has to be subtracted from the experimental abundance [6]. ESI-MS-based methods for the monitoring of ${ }^{18} \mathrm{O} /{ }^{16} \mathrm{O}$ exchange in metabolic oligophosphates, such as for the determination of the turnover of phosphoryl in nucleotide triphosphates, which occurs in many different metabolic processes, has been developed [16,17]. No LC-QTOF-MS method, however, has been developed that would allow for the PIX rates to be monitored; such a method would allow one to determine the reversibility of ATP hydrolysis by Lon proteases. In this study, therefore, we explored LC-QTOFMS as a tool for analyzing inorganic phosphate.

When compared the sample preparation required for LC-QTOF-MS analysis to GC-MS analysis of the same sample is simple and efficient, and this technique could potentially be used to monitor the PIX rates in underivatized samples. Moreover, as no derivatization agent was used for the sample preparation, no interference was expected in the interpretation of the incorporation of ${ }^{18} \mathrm{O}$. Furthermore, the use of a LC column significantly reduced the matrix effect and made the detection method significantly more sensitive than the direct flow injection analysis of the sample by LC-QTOF-MS. This new method is more sensitive than GC-MS, as its detection limit is $10.0 \mathrm{nM}$ rather than $60.0 \mathrm{nM}$.

The methodology for monitoring PIX rates using LC-QTOF-MS in enzyme-catalyzed reactions was developed in conjunction with Lon protease. Because Lon protease is known to catalyze ATP hydrolysis, in the exchange reaction in the presence of ${ }^{18} \mathrm{O}$ enriched water and in the incorporation of ${ }^{18} \mathrm{O}$ into inorganic phosphate, this enzyme proved to be suitable for testing for PIX within inorganic phosphate using LCQTOF-MS. If ATP hydrolysis is reversible, it can result in the addition of multiple ${ }^{18} \mathrm{O}$. The experimental isotopic abundance was therefore compared to the expected molecular weight distribution from naturally occurring isotopes; the results of the enzymatic hydrolysis of ATP in the presence of ${ }^{18} \mathrm{O}$-labeled water indicate that only one labeled oxygen was incorporated into any single phosphate; this was determined as there was no enrichment of any isotope except for the parent ion $+2 \mathrm{~m} / \mathrm{z}\left(\mathrm{H}_{2} \mathrm{P}^{16} \mathrm{O}_{3}{ }^{18} \mathrm{O}^{-}\right)$.

\section{Conclusion}

We have shown that the LC-QTOF-MS method that has been developed by this study can be used to measure the ${ }^{18} \mathrm{O}$ content of underivatized samples. The disadvantages of other commonly applied analytical methods like GC-MS and NMR can be overcome by this new LC-MS method. The method is fast, simple, and significantly more sensitive than the GC-MS method. It can be applied to PIX experiments to elucidate the mechanism of many enzyme-catalyzed reactions to understand the reaction rates, reaction mechanisms by evaluating the isotope enrichment without the need of derivatization. This technique was successfully applied to the measurement of positional isotope experiment rates in enzyme-catalyzed reactions so that the reversibility of the reactions could be monitored.

\section{Funding}

This work was supported by the National Science Foundation, USA. MRI Grant CHE-2923308 to YX and CHE-1213175 and CHE-1507792 I.L and I. Cheng.

\section{References}

[1] A.N. Singh, L.S. Hester, F.M. Raushel, Examination of the mechanism of sucrose synthetase by positional isotope exchange, J. Biol. Chem, 262 (1987) 2554-2557.

[2] F.C. Kokesh, Y. Kakuda, Evidence for intermediate formation in the mechanism of potato starch -hosphorylase from exchange of the ester and phosphoryl oxygens of alpha-D-glucopyranose 1-phosphate, Biochemistry 16 (1977) 2467-2473.

[3] W. Von der Saal, P.M. Anderson, J.J. Villafranca, Mechanistic Investigations of Escherichia coli Cytidine-5'- triphosphate synthetase. Detection of an intermediate by positional isotope exchange experiments, J. Biol. Chem. 260 (1985) 14993-14997.

[4] I.A. Rose, Positional isotope exchange studies of enzyme mechanisms, Advances in Enzymology and Related Areas of Molecular Biology, John Wiley \& Sons, Inc., 1979, pp. 361-395.

[5] Y. Xiao, D. Rooker, Q. You, C. Free Meyers, P. Liu, IspG-catalyzed positional isotopic exchange in methylerythritol cyclodiphosphate of the deoxyxylulose phosphate pathway: mechanistic implications, Chembiochem 12 (2011) 527-530.

[6] D.D. Hackney, K.E. Stempel, P.D. Boyer, Oxygen-18 probes of enzymic reactions of phosphate compounds, Methods Enzymol. 60 (1980) 60-83.

[7] L.S. Hester, F.M. Raushel, Analysis of ping-pong reaction mechanisms by positional isotope exchange, J. Biol. Chem. 262 (1987) 12092-12095.

[8] F.M. Raushel, L.J. Garrard, A positional isotope exchange study of the argininosuccinate lyase reaction, Biochemistry 23 (1984) 1791-1795.

[9] P.P. Dzeja, K.T. Vitkevicius, M.M. Redfield, J.C. Burnett, A. Terzic, Adenylate kinase-catalyzed phosphotransfer in the myocardium-increased contribution in heart failure, Circ. Res. 84 (1999) 1137-1143.

[10] R.J. Zeleznikar, R.A. Heyman, R.M. Graeff, T.F. Walseth, S.M. Dawis, E.A. Butz, N.D. Goldberg, Evidence for compartmentalized adenylate kinase catalysis serving a high energy phosphoryl transfer function in rat skeletal muscle, J. Biol. Chem. 265 (1990) 300-311.

[11] D. Pucar, P.P. Dzeja, P. Bast, N. Juranic, S. Macura, A. Terzic, Cellular energetics in the preconditioned state-protective role for phosphotransfer reactions captured by 180-assisted 31P NMR, J. Biol. Chem. 276 (2010) 44812-44819.

[12] D. Pucar, P.P. Dzeja, P. Bast, R.J. Gumina, C. Drahl, L. Lim, N. Juranic, S. Macura, A. Terzic, Mapping hypoxia-induced bioenergetic rearrangements and metabolic signaling by 180-assisted 31P NMR and 1 H NMR spectroscopy, Mol. Cell. Biochem. 256 (2004) 281-289.

[13] J. Thomas, J. Fishovitz, I. Lee, Utilization of positional isotope exchange experiments to evaluate reversibility of ATP hydrolysis catalyzed by Escherichia coli Lon protease, Biochem. Cell Biol. 88 (2010) 119-128.

[14] D. Vineyard, J. Patterson-Ward, I. Lee, Single-turnover kinetic experiments confirm the existence of high- and low-affinity ATPase sites in Escherichia coli lon protease, Biochemistry 45 (2006) 4602-4610.

[15] J. Fishovitz, Z. Sha, S. Chilakala, I. Cheng, Y. Xu, I. Lee, Utilization of mechanistic enzymology to evaluate the significance of adp binding to human lon protease, Front. Mol. Biosci. (2017) 00047.

[16] R. Alvarez, L.A. Evans, P. Milham, M.A. Wilson, Analysis of oxygen-18 in orthophosphate by electrospray ionisation mass spectrometry, Int. J. Mass Spectrom. 203 (2000) $177-186$.

[17] E. Nemutlu, N. Juranic, S. Zhang, L. Ward, T. Dutta, K. Nair, A. Terzic, S. Macura, P. Dzeja, Electron spray ionization mass spectrometry and 2D P NMR for monitoring $\mathrm{O} / \mathrm{O}$ isotope exchange and turnover rates of metabolic oligophosphates, Anal. Bioanal. Chem. 404 (2012) 697-706. 